\title{
Correction to: Influenza
}

\author{
Jiro Fujita
}

\section{Correction to:}

\section{J. Fujita (ed.), Influenza, Respiratory Disease Series: \\ Diagnostic Tools and Disease Managements, https://doi.org/10.1007/978-981-15-9109-9}

The book was inadvertently published with the below corrections. These changes have been updated.

In Chapter 14, the author name has been corrected from "Yosule Aoki" to "Yosuke Aoki".

In chapter 18 , the first author name in reference 10 has been corrected from "Nobuo N" to "Hirotsu N". 